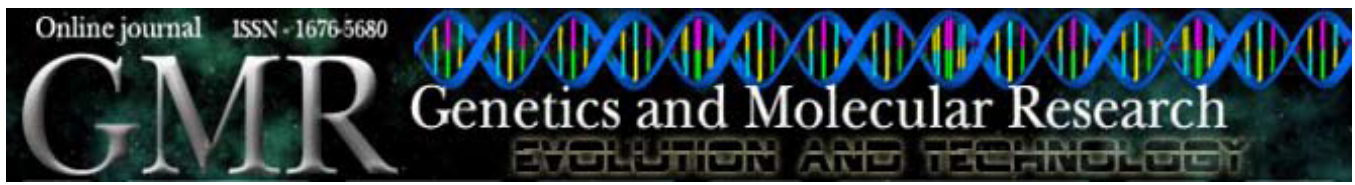

$\underline{\text { Case Report }}$

\title{
A patient with ascending aortic dilatation, similar to phenotypes of connective tissue disorders
}

\author{
S.T. Onrat ${ }^{1}$, M. Emmiler ${ }^{2}$, Y. Sıvacı ${ }^{1}$, Z. Söylemez ${ }^{1}$, \\ A. Özgöz ${ }^{1}$, N. Imirzalığlu' ${ }^{1}$ \\ ${ }^{1}$ Department of Medical Genetics, Afyon Kocatepe University, \\ Faculty of Medicine, Afyonkarahisar, Turkey \\ ${ }^{2}$ Department of Cardiovascular Surgery, Faculty of Medicine, \\ Afyonkarahisar, Kocatepe University, Afyonkarahisar, Turkey \\ Corresponding author: S.T. Onrat \\ E-mail: tutgunonrat@yahoo.com
}

Genet. Mol. Res. 8 (2): 426-434 (2009)

Received February 15, 2009

Accepted March 6, 2009

Published April 14, 2009

\begin{abstract}
We report on the clinical and molecular findings of a patient who presented alopecia, epicanthus, micrognathia, retrognathia, high arched palate, hypertelorism, Chiari type I malformation, mixedtype hearing loss but with normal heartbeat Q-T interval, malformed earlobes, down-slanted palpebral fissures, downturned corners of the mouth, syndactyly, atopic eczema, and seizures. The patient was a male adult, 23 years old, with short stature $(153 \mathrm{~cm})$ and low weight $(50.5 \mathrm{~kg})$, due to severe aortic insufficiency and dilatation of the ascending aorta. Conventional cytogenetic screening did not show any chromosomal gains or losses. Molecular genetic screening was conducted for gene mutations involved in various syndromes; the mutations found included [ $\beta$-fibrinogen $-455 \mathrm{G}>\mathrm{A}$ wt/wt (wt/mut), PAI-1 4G/5G (4G/4G), HPA1 a/b (a/a), MTHFR C677T wt/wt (wt/mut),
\end{abstract}


ACE I/D (I/I), and Apo E E3/E4]. Many clinical and molecular genetics findings overlapped with other conditions associated with arterial tortuosity and arterial aneurysms, including the Marfan, Ehler-Danlos, Shprintzen-Goldberg, and Loeys-Dietz syndromes. Although a diagnosis of Shprintzen-Goldberg syndrome was based on clinical findings and radiographic findings indicate other syndromes, aortic root dilatation seems to be a new symptom, similar to phenotypes of connective tissue disorders. The unique grouping of clinical manifestations in this patient and the molecular genetics findings lead us to suggest that this case could be an example of a previously unrecognized syndrome.

Key words: Mimicking; Ascending aorta; Connective tissue disorders; Phenotypes

\section{INTRODUCTION}

Aortic dissection is an uncommon but potentially fatal disease with catastrophic complications. A high level of suspicion is required for successful diagnosis as presenting symptoms are so variable that dissection may be overlooked in up to $39 \%$ of cases. It most commonly presents in the elderly population with a history of chronic hypertension. Rapid intervention is necessary as delay leads to higher mortality. Despite advances in diagnostic and therapeutic techniques, morbidity and mortality remain high. Advances in diagnostic imaging have raised the awareness of variants of aortic dissection, including intramural hemorrhage and penetrating aortic ulcer (Patel and Arora, 2008). Visualization of an intimal flap separating the true and false channels on morphological examinations is a pathognomonic sign of aortic dissection, confirming the positive diagnosis. In some clinical forms, such as intimo-intimal intussusception, the absence of an intimal flap in the ascending aorta can lead to delayed diagnosis and treatment. The authors have discussed the diagnostic traps and the ways of avoiding them, in the light of a case of intimo-intimal intussusception (Touati et al., 2003).

A number of genetic and environmental risk factors have been found or suspected to predispose to cardiovascular disease (CVD), a term collectively used for disorders of the heart and blood vessels. Environmental components associated with CVD include physical activity, diet, alcohol and drug consumption, smoking and stress. Genetic susceptibility may be caused by mutations and polymorphisms in a variety of genes mainly involved in blood coagulation, regulation of blood pressure, and metabolism of lipids, glucose, homocysteine, or iron. Among the candidate markers for inherited CVD risk are variations in the genes for blood coagulation factors V (FV), II (prothrombin), and XIII (FXIII), ß-fibrinogen, platelet glycoprotein IIIa (GPIIIa), plasminogen activator inhibitor-1 (PAI-1), 5,10-methylenetetrahydrofolate reductase (MTHFR), and angiotensin-converting enzyme (ACE), as well as apolipoproteins $B$ (Apo B) and E (Apo E). The most common inherited thrombophilic defect is the resistance to activated protein $\mathrm{C}$ caused by the factor $\mathrm{V}$ Leiden (FVL) mutation (a $\mathrm{G}$ to A transition at nucleotide position 1619 resulting in an Arg506Gln amino acid substitution) in 1>90\% of cases (Lane and Grant, 2000). In Caucasian populations, the prevalence of heterozygous carriers of FVL is approximately $5-7 \%$. The presence of HR2, a haplotype in the gene also encoding factor V (FVR2), has been suggested as a mild risk factor for thrombosis (Alhenc-Gelas et al., 
1999), although the polymorphic sites within FVR2 do not explain why this haplotype should alter resistance to activated protein C (Lane and Grant, 2000). Elevated plasma prothrombin and fibrinogen levels resulting from mutations/polymorphisms in factor II and fibrinogen genes (FII20210A and $\beta$-fibrinogen $455 \mathrm{G} \rightarrow \mathrm{A}$, respectively) have been considered to be associated with an increased risk for thrombosis (Lane and Grant, 2000). Furthermore, the role of a common polymorphism in the FXIII A subunit (FXIII Val34Leu), which affects cross-linked fibrin structure, as well as of a polymorphism in the promoter of the PAI-1 gene (PAI-1 4G), which results in increased plasma PAI-1 concentrations in individuals homozygous for $4 \mathrm{G}$ allele, are controversial (Catto et al., 1998; Ariens et al., 2000; Elbaz et al., 2000; Segui et al., 2000; Franco and Reitsma, 2001). The HPA-1a/1b polymorphism of human platelet antigen 1 (HPA-1), localized on platelet glycoprotein GPIIIa, has also been identified as an inherited risk factor for atherothrombosis, enhancing platelet activation (Reiner et al., 2000; Böttiger et al., 2000). Finally, two common variants (C677T and A1298C) of the gene encoding the thermolabile MTHFR have been implicated in the development of hyperhomocysteinemia, which has been identified as a risk factor for arterial and venous thrombosis (Arruda et al., 1997; Rosen, 2000).

In this study, we report on a patient affected by ascending aorta dissection and a complex clinical phenotype that does not resemble completely any of the known syndromes. This case probably represents a new syndrome. This syndrome seems to show overlap with other disorders, especially Marfan syndrome, Loeys-Dietz syndrome, Ehler-Danlos syndrome, and Shprintzen-Goldberg syndrome, for example.

\section{MATERIAL AND METHODS}

\section{Clinical data}

\section{Patient}

The patient was a male adult, 23 years old, who had been treated for epilepsy with antiepileptic drugs (Depakin, Tegretol and Keppra) in 1994. Paternal family history was unremarkable (Figure 1). He was admitted to our hospital because of ascending aortic dissection. After heparin administration, the axillary artery was cannulated with a 14-gauge femoral arterial cannula, and median sternotomy was performed. In this patient, with a two-staged single venous cannula, a cardiopulmonary bypass (CPB) was initiated and systemic cooling was accomplished. The ascending aorta was cross-clamped in its midportion. Cold-blood cardioplegia was used for myocardial preservation (blood/ crystalloid cardioplegia ratio 4:1) using a Medtronic CardioTerm ${ }^{\mathrm{TM}}$ cardioplegia system. Blood cardioplegia was infused in retrograde fashion via the coronary sinus until cardiac arrest was established. Subsequently, intermittent retrograde cold-blood cardioplegia was administered every $20 \mathrm{~min}$. A longitudinal aortotomy was made in the ascending aorta. Replacement of the aortic valve and ascending aorta with reimplantation of coronary buttons (Bentall operation) was performed with CPB. The duration of CPB was 102 min and $\mathrm{X}$-clamp was $71 \mathrm{~min}$. The core temperature of the patient was decreased down to $27^{\circ} \mathrm{C}$ during the procedure. $\mathrm{CPB}$ was terminated uneventfully and without any requirement for inotropic support. 


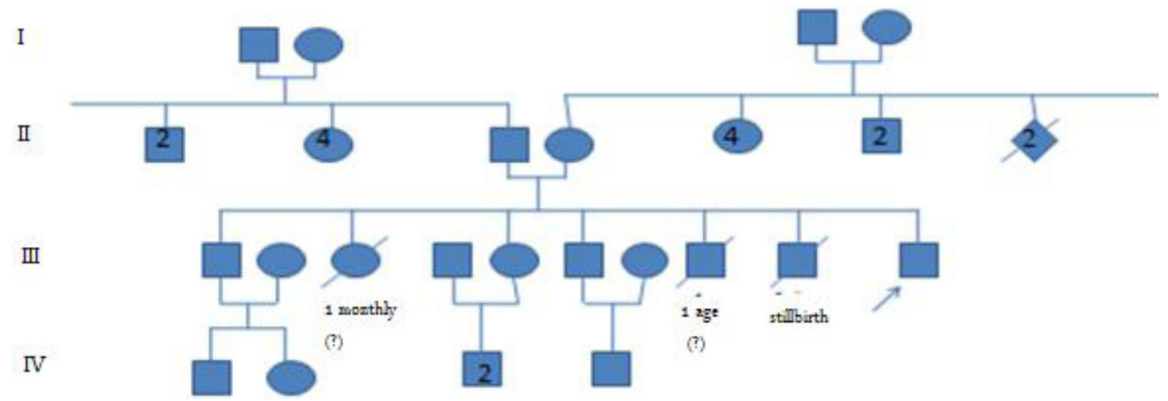

Figure 1. Patient's family pedigree.

The patient was recruited for study only when the diagnosis of ascending aortic dilatation had been established based on clinical symptoms and signs and documented with imaging technique (CT scan, MRI, MR angiography, EEG, ECO). His weight was $50.5 \mathrm{~kg}$, height 153 $\mathrm{cm}$, and he had alopecia, epicanthus, micrognathia, retrognathia, hypotelorism, Chiari type I malformation, mixed-type hearing loss but with normal heart beat Q-T interval, malformed earlobes, down-slanted palpebral fissures, downturned corners of the mouth, syndactyly, atopic eczema, seizures, hypertelorism, maxillary hypoplasia, and dilatation of the ascending aorta (Figure 2A). The facial features mainly included midfacial hypoplasia, hypertelorism with asymmetric position of the eyes, short palpebral fissures with epicanthus inversus, saddle nose, long philtrum and very small mouth with thin lips and with small low-set ears. All these facial signs suggest the presence of a distinct syndrome.

\section{A}

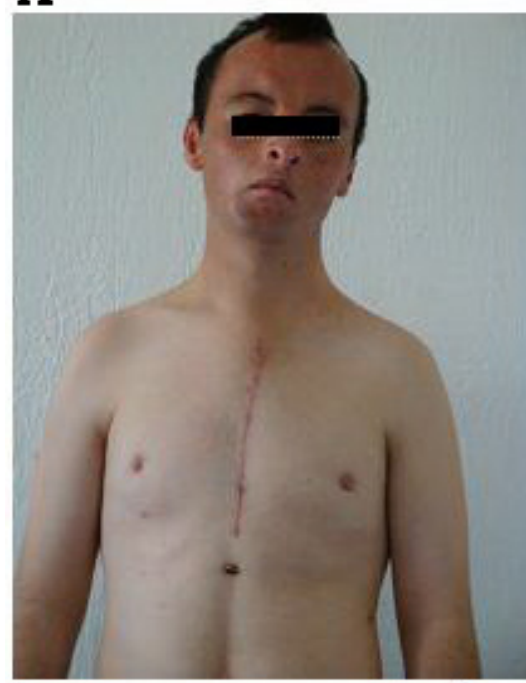

B

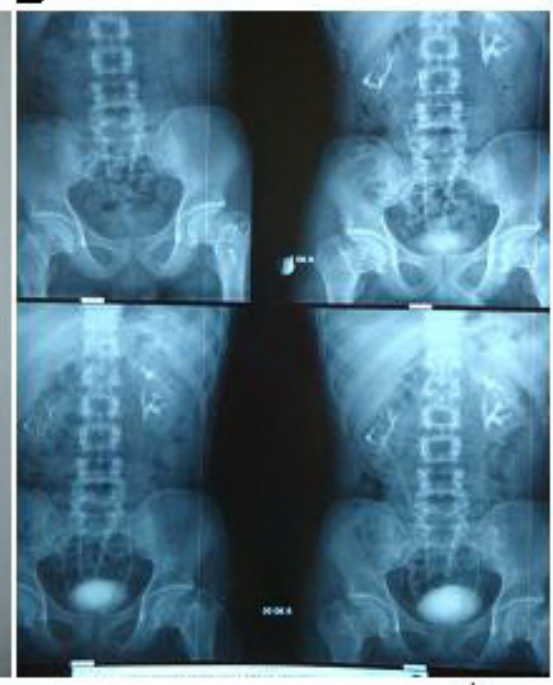

Figure 2. A. Echocardiogram showed aortic regurgitation and dilatation in the sinus area of the ascending aorta, measuring at least $5.5 \mathrm{~cm}$. A Bentall procedure was performed. B. Right kidney localization showing malrotation. 
A systolic click was audible at the lower left sternal border. Development was mildly delayed. An echocardiogram demonstrated minimal enlargement of the aortic root and mitral valve prolapse with mild tricuspid insufficiency. Echocardiogram showed aortic regurgitation and dilatation in the sinus area of the ascending aorta, measuring at least $5.5 \mathrm{~cm}$. A Bentall procedure was performed in Afyon Kocatepe University Hospitals (Figure 2A). Magnetic resonance angiography did not show abnormalities of the extracardiac large vessels.

Cranial MRI confirmed turricephaly with partial fusion of the sagittal and coronal sutures, deformation of the partially empty sella turcica, hypoplasia of the nasal bones, and zygomatic hypoplasia. Chiari I malformation and triventricular hydrocephalus were also visualized. The patient's chromosomes were normal (46,XY). Both kidneys had normal parenchymal structures in the medulla, although there was malrotation of the right kidney (Figure 2B). The patient had bilateral syndactyly between toes 2 and 3, but it did not include foot bone structures (Figure 3).

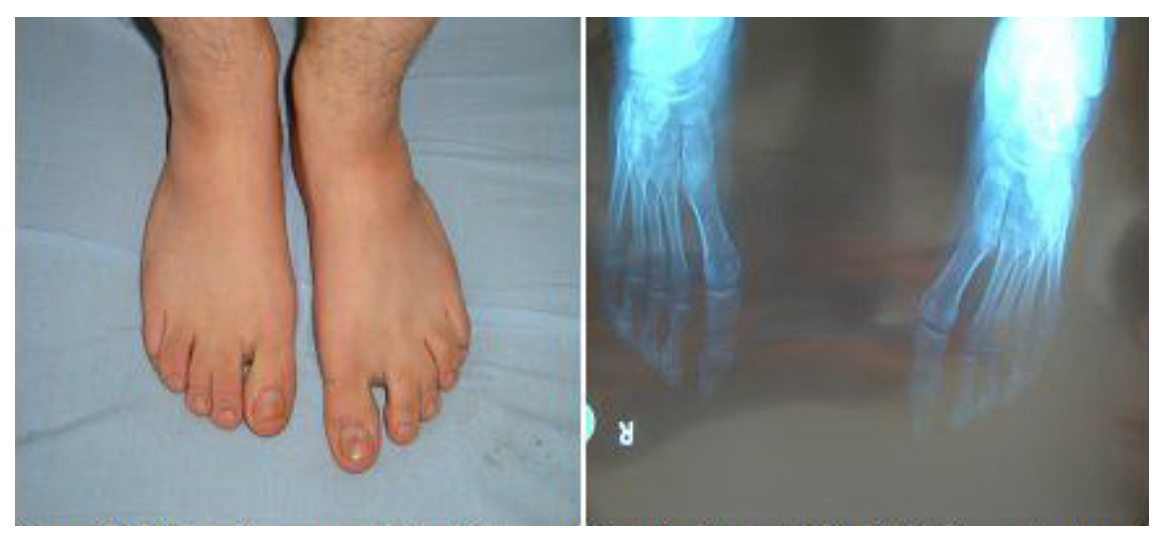

Figure 3. Bilateral syndactyly between toes 2 and 3, but not involving foot bone structures.

\section{Molecular genetics}

\section{DNA extraction and CVD strip assay}

Whole blood samples drawn from a peripheral vein into EDTA tubes were stored at $-20^{\circ} \mathrm{C}$. DNA was extracted using a commercial kit (Vienna Lab Kit) and was consequently stored at $4^{\circ} \mathrm{C}$, until used for analysis. The 12 candidate genetic risk factors for thrombosis FV R506Q (Leiden), FV H1299R (R2), prothrombin G20210A, factor XIII V34L, ß-fibrinogen -455 G-A, PAI-1 4G/5G, GPIIIa L33P (HPA-1), MTHFR C677T, MTHFR A1298C, ACE I/D, Apo B R3500Q, Apo E2/E3/E4) were also detected using a commercial kit (CVD Strip Assay; Vienna Lab, Vienna, Austria). DNA fragments including the 12 mutations/polymorphisms were amplified in vitro and biotin-labeled in two parallel multiplex polymerase chain reactions (PCR). Equal volumes $(10 \mu)$ of each PCR product and denaturating solution were mixed and incubated at room temperature for $5 \mathrm{~min}$. The alkaline reaction was neutralized with hybridization buffer, a test strip containing wild-type and mutation-specific oligonucleotide probes 
immobilized as parallel lines was added, and hybridization was carried out for $30 \mathrm{~min}$ at $45^{\circ} \mathrm{C}$ in a shaking water bath. After three stringent washes at $45^{\circ} \mathrm{C}$, bound PCR fragments were detected using a streptavidin-alkaline phosphatase conjugate and color development substrates. Upon positive reaction, a purple staining was visible after $15 \mathrm{~min}$.

The CVD Strip Assay is based on the reverse-hybridization principle, and includes three successive steps. DNA is isolated from anticoagulated blood by a rapid and convenient procedure. The relevant gene sequences are then simultaneously amplified in vitro and biotinlabeled in a single-multiplex amplification reaction. Finally, the amplification products are selectively hybridized to a test strip, which contains allele-specific (wild-type and mutant) oligonucleotide probes immobilized as an array of parallel lines.

\section{RESULTS}

Conventional cytogenetic screening did not show any chromosomal gains or losses, and the karyotype appeared normal. Furthermore, molecular genetic screening of genes involved in different syndromes, namely $\beta$-fibrinogen $-455 \mathrm{G}>\mathrm{A}$ wt/wt (wt/mut), PAI-1 4G/5G (4G/4G), HPA-1 a/b (a/a), MTHFR C677T wt/wt (wt/mut), ACE I/D (I/I), and ApoE E3/E4 genes detected mutations in genomic DNA (Table 1).

\begin{tabular}{ll} 
Table 1. Genetic results for genes tested with the CVD Strip Assay. \\
\hline Gene & Result \\
\hline Factor V G1691A (Leiden) & Homozygote normal \\
Factor V H1299R (HR2) & Homozygote normal \\
Prothrombin G20210A & Homozygote normal \\
Factor XIII V34L & Homozygote normal \\
$\beta$-fibrinogen -455 G>A & Heterozygote mutant \\
PAI-1 4G/5G & Homozygote mutant 4G/4G \\
HPA-1 a/b & Homozygote normal a/a \\
ACE I/D & Homozygote mutant I/I \\
MTHFR C677T & Heterozygote mutant \\
MTHFR A1298C & Homozygote normal \\
Apo B R3500Q & Homozygote normal \\
Apo E & Heterozygote genotype Apo E 3/4 \\
\hline PAI-1 = plasminogen activator inhibitor-1; HPA-1 = human platelet antigen 1; ACE = angiotensin-converting enzyme; \\
MTHFR = 5,10-methylenetetrahydrofolate reductase; Apo B and Apo E = apolipoproteins B and E, respectively.
\end{tabular}

\section{DISCUSSION}

In various studies on the genetic background of hemostasis in arterial thrombosis, an impressive number of polymorphisms have been claimed to influence the risk (Franco and Reitsma, 2001) for thrombus formation. Nevertheless, there are considerations regarding the complexity of the biology of hemostasis as well as limitations to the gene frequency calculations based on the heterogeneity of the population studied (Franco and Reitsma, 2001). In the present study, we found mutations in $\beta$-fibrinogen $-455 \mathrm{G}>\mathrm{A}$ wt/wt (wt/mut), PAI-1 4G/5G (4G/4G), HPA-1 a/b (a/a), MTHFR C677T wt/wt (wt/mut), ACE I/D (I/I) and ApoE E3/E4 genes in genomic DNA. FVL is the most common genetic factor associated with the risk of venous thromboembolic disease (Koster et al., 1993; Reiner et al., 2000; Franco and Reitsma, 2001). A 
relationship between FVL and arterial thrombotic disease is questioned in adults (Reiner et al., 2000). We did not determine FVL, FVR2, FII20210 polymorphism in our patient.

Fibrinogen is a $340-\mathrm{kDa}$ glycoprotein consisting of 3 nonidentical polypeptides $(\alpha, \beta$, and $\gamma$ ) linked by disulfide bonds. The 3 polypeptides are encoded by 3 fibrinogen genes clustered on the long arm of chromosome 4 (Kant et al., 1985). The cis and trans acting factors that regulate fibrinogen transcription have been extensively studied. The 3 genes contain promoter regions with TATA and CAAT boxes and a number of sequence elements that confer tissue (liver)-specific and enhanced expression. These include hepatic nuclear factor 1 (58 to the a and b genes) and IL-6-responsive elements (58 to all genes) (Figure 4) (Courtois et al., 1987; Dalmon et al., 1993). Therefore, it can be inferred that any mutations affecting the $\beta$-fibrinogen gene may have an impact on plasma fibrinogen levels. The most common polymorphism that has been studied so far is the G-455 $\rightarrow$ A polymorphism in the promoter region of the $\beta$-fibrinogen gene. In this study, we detected the mutation $\beta$-fibrinogen $-455 \mathrm{G}>\mathrm{A}$ wt/wt (wt/mut).

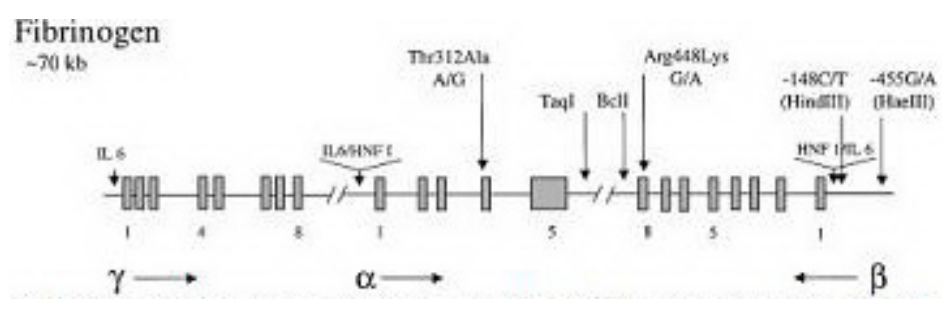

Figure 4. Representation of the organization of the fibrinogen gene locus. The 3 genes for the polypeptides are represented along with the direction in which they are transcribed (horizontal arrows). Exons (blocks) are occasionally numbered. Common polymorphisms discussed in the text are indicated by vertical arrows, as are tissue-specific (hepatic nuclear factor 1, HNF 1) and enhancer (interleukin 6, IL-6) elements.

Marfan syndrome is an autosomal dominant disorder of connective tissue caused by a mutation within the fibrillin gene located on chromosome 15 (Kainulainen et al., 1990; Dietz et al., 1991). The life-threatening cardiovascular effects of this syndrome are caused by medial aortopathy, which leads to dilatation and/or dissection of the aorta (Elkayam et al., 1995). FXIII Val34Leu polymorphism has been reported to be associated with increased plasma FXIII activity levels (Ariens et al., 2000). We did not determine FXIII Val34Leu polymorphism of this gene in our patient. On the other hand, PAI-1 regulates fibrinolysis (Franco and Reitsma, 2001), an important protective mechanism against thrombosis. Increased plasma PAI-1 levels have also been related to increased risk of arterial thrombosis, although not uniformly (Lane and Grant, 2000; Reiner et al., 2000; Franco and Reitsma, 2001). While the PAI-1 4G/5G polymorphism has been associated with increased plasma PAI-1 levels, a metaanalysis of 12 studies indicated an overall slightly increased risk of MI associated with the 4G allele (Iacoviello et al., 1998). In contrast, a protective role for $4 \mathrm{G}$ has also been suggested. We determined PAI-1 4G/4G polymorphism in our patient. Homozygosity for the 677T MTHFR variant, present in 5-20\% of Caucasians (Isotalo et al., 2000; Reiner et al., 2000), has been proposed to predispose individuals to the development of hyperhomocysteinemia. Nevertheless, a recent meta-analysis of 23 case-control studies concluded that homozygosity for the C677T MTHFR variant is not associated with arterial disease. Thus, indirectly, this absence of an association raises doubt about the role of mild hyperhomocysteinemia as a cause of 
thrombosis (Brattstrom et al., 1998). In the present study, we determined 677T MTHFR (wt/ mut) polymorphism in our patient.

In summary, our study confirmed the predominance of male thrombosis, but no definite conclusion could be reached regarding the involvement of the tested mutations/polymorphisms in the patient, except for FVL and the probably protective role of FVR2. The differentiation of the levels of their respective coded proteins implies the possibility of a more complex and multifactorial mechanism for this patient. Well-conducted studies on a larger scale should investigate the role of the genetic background of overlapping syndromes and could answer all unresolved questions in the future.

The facial features mainly included midfacial hypoplasia, hypertelorism with asymmetric position of the eyes, short palpebral fissures with epicanthus inversus, saddle nose, long philtrum and very small mouth with thin lips, and small low-set ears. All these facial signs suggest the presence of a distinct syndrome. Since no known syndromes seem to include the whole range of features of this patient and since cytogenetic screening failed to detect any chromosomal abnormality, we propose that our findings could represent a new syndrome.

In summary, according to the relationship with other syndromes, this syndrome seems like chromosome $18 \mathrm{q}$ deletion syndromes, considering the presentation of hearing loss, malformed earlobes, down-slanted palpebral fissures, downturned corners of the mouth, syndactyly, atopic eczema, seizures, short stature $(153 \mathrm{~cm})$, and poor weight gain $(50.5 \mathrm{~kg})$. In addition, this syndrome also seems like Digeorge syndrome, with short stature $(153 \mathrm{~cm})$, micrognathia, hearing loss, high arched palate in mouth, seizures, and hypernasal speech. At the same time, there is considerable clinical and molecular genetic findings overlapping with that of other conditions associated with arterial tortuosity and arterial aneurysms, including Marfan syndrome, Ehler-Danlos syndrome, Shprintzen-Goldberg syndrome and Loeys-Dietz syndrome, among other disorders. Although the diagnosis of Shprintzen-Goldberg syndrome is suspected in individuals with characteristic clinical findings and radiographic findings as in other syndromes, aortic root dilatation is most likely not found.

\section{REFERENCES}

Alhenc-Gelas M, Nicaud V, Gandrille S, van Dresden P, et al. (1999). The factor V gene A4070G mutation and the risk of venous thrombosis. Thromb. Haemost. 81: 193-197.

Ariens RA, Philippou H, Nagaswami C, Weisel JW, et al. (2000). The factor XIII V34L polymorphism accelerates thrombin activation of factor XIII and affects cross-linked fibrin structure. Blood 96: 988-995.

Arruda VR, von Zuben PM, Chiaparini LC, Annichino-Bizzacchi JM, et al. (1997). The mutation Ala $677 \rightarrow$ Val in the methylene tetrahydrofolate reductase gene: a risk factor for arterial disease and venous thrombosis. Thromb. Haemost. 77: 818-821.

Böttiger C, Kastraki A, Kah W, Mehilli J, et al. (2000). HPA-1 and HPA-3 polymorphisms of the platelet fibrinogen receptor and coronary artery disease and myocardial infarction. Thromb Haemost. 83: 559-562.

Brattstrom L, Wilcken DE, Ohrvik J and Brudin L (1998). Common methylenetetrahydrofolate reductase gene mutation leads to hyperhomocysteinemia but not to vascular disease: the result of a meta-analysis. Circulation 98: 2520-2526.

Catto AJ, Kohler HP, Bannan S, Stickland M, et al. (1998). Factor XIII Val 34 Leu: a novel association with primary intracerebral hemorrhage. Stroke 29: 813-816.

Courtois G, Morgan JG, Campbell LA, Fourel G, et al. (1987). Interaction of a liver-specific nuclear factor with the fibrinogen and alpha 1-antitrypsin promoters. Science 238: 688-692.

Dalmon J, Laurent M and Courtois G (1993). The human beta fibrinogen promoter contains a hepatocyte nuclear factor 1-dependent interleukin-6-responsive element. Mol. Cell Biol. 13: 1183-1193.

Dietz HC, Cutting GR, Pyeritz RE, Maslen CL, et al. (1991). Marfan syndrome caused by a recurrent de novo missense 
mutation in the fibrillin gene. Nature 352: 337-339.

Elbaz A, Poirier O, Canaple S, Chedru F, et al. (2000). The association between the Val34Leu polymorphism in the factor XIII gene and brain infarction. Blood 95: 586-591.

Elkayam U, Ostrzega E, Shotan A and Mehra A (1995). Cardiovascular problems in pregnant women with the Marfan syndrome. Ann. Intern. Med. 123: 117-122.

Franco RF and Reitsma PH (2001). Gene polymorphisms of the haemostatic system and the risk of arterial thrombotic disease. Br. J. Haematol. 115: 491-506.

Iacoviello L, Burzotta F, Di Castelnuovo A, Zito F, et al. (1998). The 4G/5G polymorphism of PAI-1 promoter gene and the risk of myocardial infarction: a meta-analysis. Thromb. Haemost. 80: 1029-1030.

Isotalo PA, Wells GA and Donnelly JG (2000). Neonatal and fetal methylenetetrahydrofolate reductase genetic polymorphisms: an examination of C677T and A1298C mutations. Am. J. Hum. Genet. 67: 986-990.

Kainulainen K, Pulkkinen L, Savolainen A, Kaitila I, et al. (1990). Location on chromosome 15 of the gene defect causing Marfan syndrome. N. Engl. J. Med. 323: 935-939.

Kant JA, Fornace AJ Jr, Saxe D, Simon MI, et al. (1985). Evolution and organization of the fibrinogen locus on chromosome 4: gene duplication accompanied by transposition and inversion. Proc. Natl. Acad. Sci. U. S. A. 82: 2344-2348.

Koster T, Rosendaal FR, de Ronde H, Briet E, et al. (1993). Venous thrombosis due to poor anticoagulant response to activated protein C: Leiden Thrombophilia Study. Lancet 342: 1503-1506.

Lane DA and Grant PJ (2000). Role of hemostatic gene polymorphisms in venous and arterial thrombotic disease. Blood 95: 1517-1532.

Patel PD and Arora RR (2008). Pathophysiology, diagnosis, and management of aortic dissection. Ther. Adv. Cardiovasc. Dis. 2: 439-468.

Reiner AP, Kumar PN, Schwartz SM, Longstreth WT Jr, et al. (2000). Genetic variants of platelet glycoprotein receptors and risk of stroke in young women. Stroke 31: 1628-1633.

Rozen R (2000). Genetic modulation of homocysteinemia. Semin. Thromb. Hemost. 26: 255-261.

Segui R, Estelles A, Mira Y, Espana F, et al. (2000). PAI-1 promoter 4G/5G genotype as an additional risk factor for venous thrombosis in subjects with genetic thrombophilic defects. Br. J. Haematol. 111: 122-128.

Touati G, Carmi D, Trojette F and Jarry G (2003). Intimo-intimal intussusception: a rare clinical form of aortic dissection. Eur. J. Cardiothorac. Surg. 23: 119-121. 